\title{
Tensile properties of shape memory alloy chiral honeycombs
}

\begin{abstract}
The tensile properties of hexachiral honeycomb made of shape memory alloy (SMA) material are modelled and verified experimentally at different tensile loading and ambient temperature conditions. The numerical models describe the temperature-pseudoelastic behaviour of the shape memory alloy materials constituted by Ni48Ti46Cu6 alloy. Parametric analysis on the Poisson's ratio effects over large strain deformation and temperature conditions are performed, as well as the analysis of the dependence of the honeycomb stress-strain behaviour under uniaxial loading with temperature varying through the shape memory alloy phase transformation. Chiral SMA honeycomb samples are manufactured and tested under similar environmental conditions. The experimental results are compared with the numerical one, showing good convergence in terms of trends and overall quantitative values.
\end{abstract}

Keyword: Shape memory alloy; Chiral 